\title{
Once and only once upon a time: specifying and regulating origins of DNA replication in eukaryotic cells
}

\author{
John F.X. Diffley \\ Imperial Cancer Research Fund, Clare Hall Laboratories, South Mimms, Herts EN6 3LD, UK
}

A distinguishing feature of eukaryotic cells is the great variability they exhibit in both genome size and organization. For example, the budding yeast Saccharomyces cerevisiae contains little more DNA per cell than the prokaryote Escherichia coli, whereas cells from many plants such as the lily, Lilium longiflorum, contain nearly $10^{5}$ times as much DNA per cell. In addition to being large, eukaryotic genomes are also fragmented: DNA can be contained on just a few chromosomes or can be distributed on literally thousands of individual chromosomes. The evolution of a mechanism to rapidly replicate large amounts of DNA on multiple chromosomes was probably an important prerequisite to the expansion of genome size and consequently to the explosion of morphological and functional diversity seen among eukaryotes.

One of the most important features of this mechanism is that bidirectional DNA replication initiates from multiple origins on eukaryotic chromosomes (Cairns 1966; Huberman and Riggs 1966, 1968). While budding yeast contains only about four times the amount of DNA per haploid cell as E. coli, it replicates its genomic DNA from 250-400 replication origins distributed on 16 chromosomes, whereas the $E$. coli genome, contained on a single chromosome, is replicated from a single replication origin (oriC). Thus, although eukaryotic genomes are generally larger than prokaryotic genomes, the amount of DNA replicated from an individual replication origin is generally considerably smaller. This can allow very rapid replication. Even under optimal growth conditions, the $E$. coli genome takes $\sim 40 \mathrm{~min}$ to replicate. Drosophila melanogaster cells contain almost 100 times as much DNA as E. coli, yet in cells of early Drosophila embryos, the DNA synthetic phase of the cell cycle (S phase) takes only 3-4 min because replication initiates synchronously from many origins spaced at short (roughly 8-kb) intervals (Blumenthal et al. 1973).

Perhaps equally striking is that the number of origins used to replicate eukaryotic genomes can vary from cell type to cell type within an organism. This can allow a great deal of flexibility in the length of $\mathrm{S}$ phase. For example, although $S$ phase in Drosophila embryos is 3-4 min, it takes nearly $10 \mathrm{hr}$ for the same process to occur in cells from adult animals grown in tissue culture (Blumenthal et al. 1973). This increase in the length of $\mathrm{S}$ phase is partly attributable to a reduction in the number of origins used. In addition, origin firing in these tissue culture cells becomes highly asynchronous so that some parts of the genome replicate much earlier than others.

The firing of multiple replication origins throughout $S$ phase also presents a potential logistical nightmare; as $S$ phase progresses, origins within unreplicated DNA must be located and fired against the background of an increasingly large pool of replicated DNA. Furthermore, within a single $S$ phase, DNA replication must never reinitiate from an origin that has already fired. In this review I will summarize some of the important discoveries and concepts that have led to our current understanding of how replication origins are specified and how multiple initiation events are regulated to ensure efficient and precise duplication of the genome.

Origin specification: the role of cis-acting sequences in initiation

\section{The Replicon model and its successes}

Much of our current thinking about DNA replication has been shaped by the Replicon model of Jacob, Brenner, and Cuzin (Jacob et al. 1963). In this model, an initiator protein acts through a cis-acting sequence, the replicator, to drive the initiation of replication. This simple idea has been extremely useful in providing a framework to explain how the initiation of DNA replication occurs in a number of systems (for review, see Stillman 1993).

The Replicon model was proposed to explain the regulation of $E$. coli DNA replication. We now know that the essential initiator protein in E. coli is DnaA, which binds specifically to the replicator, oriC. On the basis of their work in E. coli, Bramhill and Kornberg proposed an important extension of the Replicon model (Bramhill and Kornberg 1988) in which the initiator protein serves three different and essential functions: (1) origin recognition; (2) induction of local unwinding within the origin; and (3) recruitment of other replication enzymes by protein-protein interactions (Fig. 1). In the case of E. coli DnaA specifically recognizes the 9 -mer and 13 -mer se- 


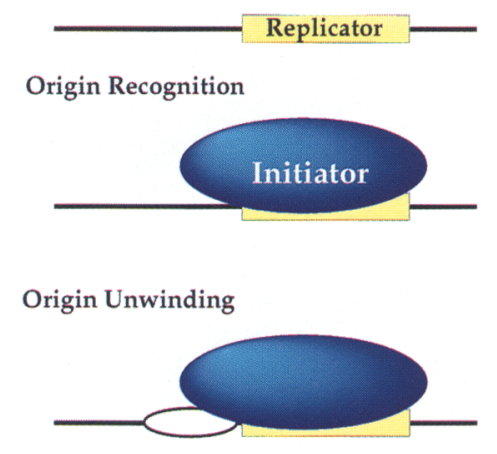

Recruitment of Replication Machinery

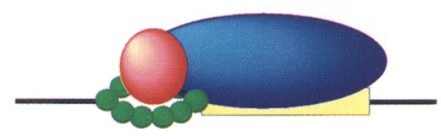

Figure 1. Roles of initiator proteins. The three roles of initiator proteins (blue) are outlined (Bramhill and Kornberg 1988). These include recognition of the replicator sequence (yellow), origin unwinding, and recruitment of components of the replication machinery (red and green). Details are discussed in the text.

quences within oriC, causes localized unwinding within the 13-mer sequences, and recruits the DnaB helicase by virtue of the mutual interactions that DnaA and DnaB have with a third protein, DnaC.

In addition to being useful in understanding initiation from a variety of prokaryotic origins, these concepts have also been useful in understanding initiation in several eukaryotic viruses, notably, the papovavirus, SV40 (Kelly 1988; Challberg and Kelly 1988; Stillman 1989; Borowiec et al. 1990). During the initiation of SV40 DNA replication, the viral encoded initiator, large $\mathrm{T}$ antigen (TAg), specifically recognizes sequences within the core origin, induces unwinding within the origin, and recruits other replication proteins such as DNA polymerase $\alpha$-primase and the single-stranded DNA-binding protein RP-A by direct protein-protein interaction (for review, see Diffley 1992).

A concept that has emerged from these studies is that the initiation of DNA replication is a multistep process in which many critical and biochemically discernible events occur at origins before the incorporation of the first templated nucleotides. This concept has been crucial for understanding eukaryotic chromosomal DNA replication.

\section{Yeast replicators}

A major advance in the study of DNA replication came in 1979 with the identification of autonomously replicating sequences (ARSs) in budding yeast (for review, see Campbell and Newlon 1991; Diffley 1995). ARSs are genomic DNA sequences that can confer on plasmids the ability to transform yeast at high frequency. In such transformants, ARS-containing plasmids are maintained as unrearranged, extrachromosomal elements, arguing that ARSs act by providing a replicator function to the plasmid. In 1987, two groups, using two-dimensional gel techniques to examine replication intermediates, demonstrated that bidirectional DNA replication actually initiates specifically within or very close to these ARS elements (Brewer and Fangman 1987; Huberman et al. 1987). It has been subseqently shown that many but not all ARSs act as replication origins even in their normal chromosomal location (for review, see Diffley 1995). Therefore, ARSs act as both genetically defined replicators and biochemially defined origins of DNA replication. The existence of such sequences provided strong support for the notion that the Replicon model might be helpful in understanding the initiation of DNA replication in eukaryotic chromosomes as well.

The sequences important for DNA replication within a number of yeast ARSs have been analyzed in some detail. All yeast ARSs contain an exact or very close match to an 11-bp ARS consensus sequence (ACS) and, in all cases tested, an ACS is essential for origin function. An ACS by itself, however, is not sufficient for origin function, and, in all cases tested, a flanking sequence is also essential for origin function. This flanking sequence is generally composed of several sequence modules that each contribute to origin function. Detailed discussion of the role of these flanking sequences is beyond the scope of this review, and the reader is referred to other sources (Marahrens and Stillman 1992; Rao et al. 1994; Theis and Newlon 1994; Huang and Kowalski 1996) for more discussion.

\section{Random initiation in early embryos}

Optimism about the usefulness of the Replicon model to describe eukaryotic DNA replication was dealt a serious blow by the experiments of Harland and Laskey (1980), who showed that a variety of DNA molecules derived from either animal viruses or prokaryotes could replicate efficiently and in a strict once-per-cell-cycle manner when microinjected into eggs of the South African clawed toad, Xenopus laevis. These experiments have had profound implications. Most importantly, these experiments argued that specific DNA sequences are not required either for initiating DNA replication or for preventing rereplication within a single cell cycle in early Xenopus embryos. To put this another way, virtually any sequence in the genome might potentially act as a replication origin. Therefore, only a small subset of potential origins is used within any single $S$ phase. Consequently, reinitiation is not only blocked from origins that have already fired, but initiation is also blocked from all of the potential, unfired origins within regions of replicated DNA. This has led to the important idea that the act of replicating a DNA sequence renders it unable to support initiation until the next cell cycle (for review, see Harland 1981).

Studies on Xenopus DNA replication have also been important because they have provided one of the only cell-free systems for studying eukaryotic chromosomal 
DNA replication. When incubated in an extract from Xenopus eggs, sperm chromatin or purified DNA are first assembled into nuclei and subsequently replicated (Lohka and Masui 1983; Blow and Laskey 1986). This system appears to faithfully reflect events occurring in vivo as replication of input molecules occurs just once (Blow and Laskey 1986). As is the case in vivo, a number of different DNA molecules that do not contain any eukaryotic sequences, such as bacteriophage $\lambda$ DNA, replicate in a regulated manner in this system. Application of the same origin mapping techniques used to show specific initiation in yeast at ARSs revealed apparently random initiation on input plasmid molecules in these Xenopus extracts (Hyrien and Mechali 1992; Mahbubani et al. 1992), consistent with the idea that any sequence can act as a replication origin in early Xenopus embryos.

This lack of requirement for specific replicator sequences is not unique to Xenopus but is seen in at least some other early metazoan embryos. A cell-free system conceptually similar to the Xenopus system described above has been developed with extracts from early Drosophila embryos (Crevel and Cotterill 1991). In this system, as in Xenopus, exogenously added DNA is first assembled into nuclei and subsequently replicated. Again, as in Xenopus extracts, specific eukaryotic sequences are not required for regulated DNA replication.

The apparently fundamental difference between replicators in yeast and metazoan embryos has presented some differences in trying to understand how DNA replication initiates in eukaryotes. As we shall see, studies on yeast and Xenopus have begun to converge during the past several years, suggesting that this paradox may be more superficial than initially thought.

\section{Specific replicators in higher eukaryotes}

The apparent lack of sequence requirement for Xenopus replicators may not be a universal feature of higher eukaryotes. Physical mapping of replication start sites has shown that initiation is not random in mammalian chromosomes and this type of analysis has led to the identification of a number of mammalian replication origins (Depamphilis 1993). The distinction between origins as biochemically defined replication start sites and replicators as genetically defined sequence elements required for initiation becomes critical here because, although mammalian replication origins have been identified, there is little genetic data indicating that these sites contain DNA sequences that are essential for replication. The one clear exception to this occurs at the $\beta$-globin locus where an origin of bidirectional DNA replication has been mapped by several techniques to a region between the $\delta$ - and $\beta$-globin genes (Kitsberg et al. 1993; Aladjem et al. 1995). Initiation no longer occurs in this region in cells derived from a patient suffering from $\beta$-thalassemia in which an 8 -kb sequence of DNA containing the $\beta$-globin origin has been removed (Kitsberg et al. 1993). In these cells, the $\beta$-globin locus is replicated passively from an uncharacterized origin outside of the $\beta$-globin locus. Presumably, then, some DNA sequence within this region is essential for specifying an origin at this location.

The $\beta$-globin origin is active in both erythroid and nonerythroid cell types even though it lies within a cluster of genes expressed only in erythroid cells. Expression of the globin genes requires the locus control region (LCR), a sequence $>50 \mathrm{~kb}$ upstream from the $\beta$-globin gene. The LCR also appears to be essential for the firing of the $\beta$-globin origin because the $\beta$-globin locus is replicated passively from an origin outside of the $\beta$-globin locus in mutants where the LCR has been deleted (Aladjem et al. 1995). The LCR, in addition to its role in transcriptional regulation, therefore, also has an essential role in DNA replication even when the $\beta$-globin locus is transcriptionally inactive. These results indicate that sequences located well outside of a biochemically defined origin can be essential for that origin to fire. Thus, isolation of active replicators from mammalian cells by use of assays similar to the yeast ARS assay that require the cloning of putative replicators onto plasmids may prove difficult, as large regions of DNA may be required for origin activity. This requirement may help to explain the difficulty in identifying mammalian replicators by this approach (for discussion, see Krysan et al. 1993; Aladjem et al. 1995).

\section{ORC as a conserved eukaryotic initiator}

\section{Budding yeast ORC}

The existence of the essential ACS in budding yeast replication origins suggested that a protein might exist that would specifically recognize this sequence. After much searching, a strong candidate emerged in 1992 when Bell and Stillman (Bell and Stillman 1992) described the biochemical characterization of the Origin Recognition Complex (ORC). As its name implies, OR $\bar{C}$ is a tight complex of six polypeptides that binds to the ACS of all origins tested to date. Furthermore, there is a strong correlation between mutations within the ACS that eliminate ARS function in vivo and those that eliminate ORC binding in vitro.

In a parallel study using genomic footprinting at nucleotide resolution to examine proteins bound at the yeast replication origin ARS1 in vivo, the ACS was shown to be protected from nuclease cleavage, suggesting that it was, indeed, bound by a protein (Diffley and Cocker 1992). Furthermore, the footprint over the ACS in chromatin was very similar to that produced by purified ORC in vitro, arguing that ORC binds the ACS in vivo. Because these genomic footprints were performed on chromatin from logarithmically growing cells and the footprint over the ACS was nearly saturated, these results suggested that ORC must be bound at ARS1 during a large fraction of the cell cycle.

The genes encoding all six ORC subunits have now been isolated, and all six are essential for viability in yeast (Bell et al. 1993, 1995; Foss et al. 1993; Li and Herskowitz 1993; Micklem et al. 1993; Loo et al. 1995; Hardy 1996; Hori et al. 1996). A few of the subunit-en- 
coding genes have been analyzed in more detail and all of the available evidence indicates that ORC is essential for initiating DNA replication. For example, orc2, orc3, and orc5 mutants show cell-cycle aberration consistent with defects in DNA replication, and lose ARS-containing plasmids at a high frequency (Foss et al. 1993; Micklem et al. 193; Fox et al. 1995; Loo et al. 1995; Hardy 1996). Additionally, direct examination of replication intermediates in both orc 2 and orc 5 mutants has indicated reduced initiation frequency from chromosomal origins (Liang et al. 1995; Loo et al. 1995).

Taken together, these results argue that ORC fulfills the criteria for an initiator protein in yeast DNA replication: It specifically recognizes replicators both in vitro and in vivo, and it is itself essential for initiating DNA replication. The genomic footprinting experiments, however, suggested that ORC is bound during most or all of the cell cycle (Diffley and Cocker 1992). Subsequent genomic footprinting experiments showed that ORC remains bound at origins afer DNA replication during the $\mathrm{G}_{2}$ and $\mathrm{M}$ phases of the cell cycle (Diffley et al. 1994). Therefore, the binding of ORC to the ACS, although essential, is not sufficient to drive the initiation of DNA replication in vivo.

\section{ORC in Xenopus, Drosophila, and beyond}

It was of considerable interest to know whether early Xenopus or Drosophila embryos, which do not require specific replicators, use an initiator protein related to the yeast ORC. A beginning to the resolution of this issue has been obtained recently with the isolation of several ORC subunit homologs in other eukaryotes (Ehrenhofer Muray et al. 1995; Gavin et al. 1995; Gossen et al. 1995; Muzi-Falconi and Kelly 1995; Carpenter et al. 1996; Leatherwood et al. 1996; Rowles et al. 1996). ORC1- and ORC2-related proteins have been found in Xenopus egg extracts (Carpenter et al. 1996; Rowles et al. 1996) and ORC2- and ORC5-related proteins have been found in early Drosophila embryos (Gossen et al. 195). Morever, multisubunit complexes containing these proteins have been isolated from both Xenopus and Drosophila, strongly suggesting that an ORC-like complex is present during early metazoan development. An obvious possibility is that the other polypeptides in these complexes are homologs of the other yeast ORC subunits.

The availability of cell-free systems for DNA replication has made functional studies possible. Immunodepletion with antibodies to either ORC1 or ORC2 from Xenopus egg extracts renders these extracts unable to initiate DNA replication (Carpenter et al. 1996; Rowles et al. 1996). Replication ability of ORC2-depleted extracts is not restored by addition of recombinant ORC2 alone; however; the replication ability of either ORC1or ORC2-depleted extracts can be restored by addition of protein fractions containing the multisubunit ORC. These experiments indicate that an origin recognition complex is not only present, but also appears to be essential for DNA replication during early development. That specific replicator DNA sequences are not required for Xenopus or Drosophila replication might have suggested that the basic mechanism of initiation might be different in yeast and frogs; however, the finding that a conserved initiator protein is required makes this far less likely.

Human homologs of ORCl and ORC2 have also been isolated (Gavin et al. 1995). ORC-like complexes containing these proteins have not yet been isolated from human cell extracts, but this clearly represents an important goal for the future. Convergence of this line of research with characterization of replicators such as that found in the $\beta$-globin locus described above should provide important details about the role of ORC in replicator function and origin specification in nonembryonic cells from higher eukaryotes.

\section{Possible resolutions of the replicator specificity paradox}

The experiments described above suggest that ORC may act as an initiator protein in all eukaryotic cells. This does not resolve the replicator specificity paradox but only serves to heighten it. How can a protein which acts to bind specific DNA sequences and consequently specify origin location in budding yeast also have an essential role in sequence-independent initiation in early embryos? Some scenarios that might explain this paradox are considered below.

One possibility is that, because of evolutionary pressure to accelerate the rate of early embryogenesis, metazoans have evolved a special embryonic form of ORC that does not exhibit sequence specificity in its DNA binding, allowing initiation events to occur randomly and at a high density along chromosomes. This form of ORC might only be present during early development. Later in development and in the adult, a sequence-specific form of ORC might be present that only interacts with specific replicators such as the $\beta$-globin sequences described above.

A second possibility is that $O R C$ is a sequence-specific DNA-binding protein, but it is present in such high concentrations in early embryos that lower affinity nonspecific DNA binding or binding to very degenerate sequences allows initiation to occur apparently randomly. Such a model would not be inconsistent with biochemical analysis of yeast ORC. In the presence of nonspecific competitor DNA, ORC exhibits very strong sequence preference, requiring both an intact ACS as well as an adjacent sequence element known as B1 for efficient binding (Rao and Stillman 1995; Rowley et al. 1995). In the absence of competitor DNA, however, ORC no longer requires the $\mathrm{B} 1$ element for specific binding. Moreover, under these conditions, ORC will bind specifically to a degenerate $(9 / 11)$ match to the ACS found within ARS1 when the ACS has been eliminated by mutation. Thus, even yeast ORC might have the capability to bind to very degenerate sequences at sufficiently high concentrations.

Finally, metazoan ORC in both early embryos and in adults might be a nonspecific DNA-binding protein. In 
this model, the apparent origin specificity seen in adult cells would come not from sequence specificity of ORC itself, but from other features of chromosomes. Specificity might come from other sequence-specific DNA-binding proteins such as transcription factors that might only be required for replication in nonembryonic cells when concentrations of initiation factors such as ORC are lower. Alternatively, the apparent origin specificity seen in mammalian cells may not be a result of active choice of origins, but, rather, may be largely a result of exclusion of initiation from various regions of chromosomes such as those being actively transcribed. Origins in both yeast and higher eukaryotes are generally found in intergenic regions, not within genes, and transcription through origins inactivates them in both yeast and human cells (Snyder et al. 1988; Haase et al. 1994; Tanaka et al. 1994). Early Xenopus embryos are transcriptionally inactive, which may be relevant in explaining the apparently random initiation, and it has been shown that the transition from nonspecific to specific initiation within the rDNA locus occurs at approximately the time this locus becomes transcriptionally active /Hyrien et al. 1995). It is important to note that in nonerythroid cells, initiation within the $\beta$-globin locus is still localized to the $\delta-\beta$-globin intergenic region, indicating that transcriptional inactivity of a region is not sufficient to cause random initiation across it.

The possibilities outlined above are, of course, not necessarily mutually exclusive. For example, ORC in higher eukaryotes might have marginal sequence specificity and might also be excluded from transcribed regions. Further work on the biochemical properties of ORC from higher eukaryotes should help to resolve this issue.

\section{Two steps in regulating initiation}

As discussed above, genomic footprinting in budding yeast has indicated that the binding of ORC is not sufficient to drive the initiation of DNA replication and, therefore, initiation must be regulated in other ways. Studies of yeast and Xenopus replication have again begun to converge to provide a model for how DNA replication is regulated in eukaryotes to ensure efficient once-per-cell-cycle replication. At the heart of this model is a simple two-step mechanism in which the assembly of stable prereplicative complexes (pre-RCs) at origins precedes and is temporally separated from the firing of these origins. Regulation of this process by cyclin-dependent kinases ensures that when origins fire, the cell is no longer competent to form new pre-RCs, thus limiting replication to once per cell cycle. Some of the details of this mechanism are considered below.

(Step 1) Resetting nuclei: competence to assemble pre-RCs at origins

Much of our understanding of global cell-cycle control comes from early cell fusion and nuclear transplantation experiments. In a classic series of experiments by Rao and Johnson (1970), HeLa cells synchronized at different points in the cell cycle were fused, and the effect of the fusion on the individual nuclei of the heterokaryon was examined. When $S$ phase and $G_{1}$ phase cells were fused, the $G_{1}$ nuclei entered $S$ phase prematurely. This demonstrated that $S$ phase cells contain diffusible $S$ phase-promoting factor(s) essential for DNA replication and that $G_{1}$ nuclei are competent to respond to these factors. However, when $S$ phase and $G_{2}$ phase cells were fused, the $G_{2}$ nuclei did not enter $S$ phase, whereas DNA synthesis in the $S$ phase nuclei was unaffected. Therefore, $\mathrm{G}_{2}$ nuclei have lost the ability to respond to the $S$ phasepromoting factors. These experiments define two different levels of control of entry into $S$ phase. First, there is a nuclear autonomous resetting event that occurs with the conversion of $G_{2}$ to $G_{1}$ nuclei and, therefore, correlates with the passage through mitosis. Second, S phasepromoting factors accumulate during late $G_{1}$ and trigger the entry of competent nuclei into Sphase.

Licensing in Xenopus The discovery that specific replicator sequences were not required for Xenopus replication led to the consideration of models to explain how rereplication might be prevented in this system. These models incorporated a common theme, namely that passage of a replication fork across a region of DNA renders this region unable to support new initiation (Harland and Laskey 1980; Harland 1981). This could be either because replication forks deposit an inibitor of initiation or remove an activator of initiation. This second idea was preferred and the replication activator was termed a "license." In this model, the license was envisioned to be "issued" at the start of S phase. This early model provided us with important concepts; however, because there was no temporal separation between licensing and origin firing, it was unclear why replicated DNA could not be relicensed.

Blow and Laskey (1988) proposed an important extension of the licensing factor model on the basis of experiments showing that treatments that transiently permeabilized the nuclear envelope allow replicated nuclei to rereplicate. In this model, licensing factor was proposed to be an essential initiation factor, which, as in the earlier model, is chromatin bound, can support only a single initiation event, and is canceled by passage of a replication fork through it. The novel feature of this model is that licensing factor can gain access to chromatin during mitosis only when the nuclear envelope breaks down, presumably because it lacks a functional nuclear localization signal during interphase. This was important for two reasons. First, by dividing initiation into two temporally separate and mutually exclusive steps, it clearly provided a potential mechanism by which rereplication within a single cell cycle could be blocked. Second, because mitosis is the only time in the cell cycle when the nuclear envelope breaks down, it provided a potential rationale for the earlier cell-fusion experiments that indicated that passage through mitosis was required for a new round of DNA synthesis. 
One drawback of this model is that it does not provide a universal mechanism for control of DNA replication in eukaryotes, as most unicellular eukaryotes undergo a closed mitosis in which the nuclear envelope does not break down, and many cell types in higher eukaryotes can undergo multiple rounds of DNA replication without intervening mitosis. Although one can invoke other mechanisms, such as mitosis-specific nuclear import of licensing factor, to reconcile this, it implies the evolution of two very different mechanisms for coupling mitosis and $\mathrm{S}$ phase. A second drawback of the model is that, if mitosis is the only time licensing can occur, it requires that the license must have almost infinite stability on chromatin, because many cells exit the cell cycle between mitosis and the onset of $S$ phase for great lengths of time. Although this is not a problem in early embryos, which are undergoing rapid cell cycles, it is an important consideration for most other eukaryotic cells.

Several labs have shown recently that proteins known collectively as the $\mathrm{Mcm}$ (minichromosome maintenancel proteins are essential for the licensing reaction (Chong et al. 1995; Kubota et al. 1995; Madine et al. 1995a). The $\mathrm{mcm}$ mutants were initially identified in budding yeast as mutants that decrease the ability of cells to maintain ARS-containing plasmids (Maine et al. 1984). Several mutants were also independently isolated as conditional lethal cell division cycle $(c d c)$ mutants that arrested with a terminal phenotype consistent with a role in DNA replication (Moir et al. 1982; Hennessy et al. 1991). In fission yeast a number of Mcm-encoding genes were also identified in genetic screens for mutants with cell-cycle defects (Coxon et al. 1992; Miyake et al. 1993; Forsburg and Nurse 1994; Takahashi et al. 1994). In budding yeast, there are six $\mathrm{Mcm}$ proteins. Five had been initially isolated as mutants as described above: Mcm2, Mcm3, Cdc46, Cdc47, and Cdc54 (Hennessey et al. 1991; Yan et al. 1991; Dalton and Whitbread 1995; Whitbread and Dalton 1995). The sixth Mcm encoding gene was identified from the genome sequencing project (unpubl.) A large number of $\mathrm{Mcm}$ homologs have been found in higher and lower eukaryotes, and phylogenetic analyses indicate that in the eukaryotic kingdom, $\mathrm{Mcm}$ proteins fall into six subfamilies (for review, see Chong et al. 1996). In budding yeast, there is a single representative from each subfamily. The function of each of the subfamilies is apparently nonredundant, as each of the well-characterized budding and fission yeast $\mathrm{Mcm}$ proteins is individually essential for viability. There is considerable evidence in yeasts and higher eukaryotes that the $\mathrm{Mcm}$ proteins interact with each other and may act as an as-yet-undefined oligomeric complex. Although no biochemical activity has yet been ascribed to the $\mathrm{Mcm}$ proteins, sequence analysis suggests that they may be members of a larger superfamily of DNA-dependent ATPases (Koonin 1993).

Pre-RCs in budding yeast Pre-RCs were identified in budding yeast by use of genomic footprinting of replica- tion origins. These complexes appear as extended regions of nuclease protection adjacent to the ACS (Diffley et al. 1994), suggesting that they involve the recruitment of additional factors to origins. Although the protein components of pre-RCs remain to be determined, recent experiments have shed some light on how these complexes are regulated. Pre-RCs assemble at replication origins at the end of mitosis in rapidly cycling cells and remain at origins throughout $\mathrm{G}_{1}$. Pre-RCs are still present at origins in cells arrested with the $c d c 7$ mutation, which arrests cells at the $G_{1}-S$ transition, but have disappeared from origins in cells blocked in $S$ phase either with inhibitors of elongation, such as hydroxyurea, or with conditional mutants in the replication fork machinery. Therefore, the disappearance of pre-RCs corresponds very closely with the initiation of DNA replication. These results led to the sugges tion that pre-RCs might play a role in initiating DNA replication.

The Cdc6 protein is essential for an early event in S phase (Hartwell 1976) and cdc6 temperature-sensitive mutants are defective in initiating DNA replication (Hogan and Koshland 1992; Liang et al. 1995). The presence of the wild-type $C D C 6$ gene on a multicopy plasmid suppresses the initiation defect in orc5 mutants, and the Cdc6 protein (Cdc $6 \mathrm{p}$ ) can interact with ORC in vitro (Liang et al. 1995), suggesting that Cdc6p and ORC might interact in vivo. By use of strains in which $C D C 6$ was under the control of a regulatable promoter, it was shown that de novo synthesis of Cdc6p after mitosis is required for pre-RCs to form. Moreover, preformed pre$\mathrm{RCs}$ are thermolabile in $c d c 6$ temperature-sensitive mutants, demonstrating that Cdc $6 \mathrm{p}$ is required for the assembly and maintenance of pre-RCs (Cocker et al. 1996). Because Cdc6p has an essential role in DNA replication, these results are consistent with the idea that pre-RCs are bona fide preinitiation complexes.

The Cdc6p homolog in the fission yeast Schizosaccharomyces pombe is the product of the $c d c 18^{+}$gene (Kelly et al. 1993). Both Cdc6p and the $c d c 18^{+}$gene product are unstable proteins (Nishitani and Nurse 1995; Piatti et al. 1995; Muzi-Falconi et al. 1996) and de novo Cdc6p and $\operatorname{cdc} 18^{+}$protein synthesis is required in each cell cycle for DNA replication to occur (Kelly et al. 1993; Piatti et al. 1995). Rather than arrest in $G_{1}$ with a $1 C$ DNA content, however, cells deprived of Cdc $6 \mathrm{p}$ or $c d c 18^{+}$protein undergo a haploid mitosis. Cells quickly loss viability in the absence of these proteins. The loss of viability of cells lacking Cdc6p, however, is not suppressed by the blocking of mitosis with the microtubule inhibitor nocodazole (L. Drury and J. Diffley, unpubl.) or by depletion of mitotic cyclins (Piatti et al. 1995). This suggests that the major cause of death is not the loss of ploidy caused by the haploid mitosis, but, rather, that Cdc6p cannot execute its essential function late in the cell cycle. The relevance of this point is discussed below.

In actively cycling cells. Cdc6p is made at the mitosis$\mathrm{G}_{1}$ boundary, roughly when pre-RCs are formed (Zwerschke et al. 1994; Piatti et al. 1995). However, when $G_{1}$ phase is extended either by the arrest of cells with mating pheromone or by growth of cells in a poor carbon 
source, Cdc $6 p$ is also made at the end of $G_{1}$, and this late $G_{1}$ expression can be important for S-phase progression (Piatti et al. 1995). This is probably because origins lose pre-RCs when cells exit the cell cycle from $G_{1}$ in poor growth conditions (Diffley et al. 1994) and reform them when they reenter the cell cycle (Cocker 1995). This has an important implication. Although pre-RCs may form at the end of mitosis in rapidly cycling cells, it is not the only opportunity cells have to form these complexes. Therefore, although passage through mitosis may be a prerequisite for pre- $\mathrm{RC}$ formation, pre- $\mathrm{RC}$ formation is not directly coupled to any biochemical process that occurs during mitosis.

Making the connection During the licensing reaction in Xenopus, Mcm proteins become associated with a nuclear structure suggested to be chromatin (Chong et al. 1995; Kubota et al. 1995; Madine et al. 1995a). Little is known about what these proteins bind to; however, this loading reaction requires factors in addition to $\mathrm{Mcm}$ proteins (Chong et al. 1995; Madine et al. 1995a). Recent experiments have indicated that at least some of these factors are also components of the yeast pre-RC pathway. One of these factors appears to be ORC, as immunodepletion with antibodies to either ORCl or ORC2 not only blocks replication, it also blocks the deposition of Mcm proteins (Coleman et al. 1996; Rowles et al. 1996). A second factor is the Xenopus homolog of Cdc6p, which has recently been isolated and shown by immunodepletion to be essential for DNA replication /Coleman et al. 1996). Moreover, extracts from which this Cdc6p homolog has been depleted can no longer support the loading of $\mathrm{Mcm}$ proteins. Xenopus Cdc6p itself appears to bind to chromatin, and this binding is blocked by immunodepletion of ORC. In budding yeast the $\mathrm{Mcm}$ proteins are also bound to chromatin during $G_{1}$ (Yan et al. 1993; S. Donovan and J. Diffley, unpubl.) By use of the same approaches used to show the essential role for Cdc6p in pre-RC formation, it has been shown recently that de novo Cdc6p synthesis is reqired for the chromatin binding of budding yeast $\mathrm{Mcm}$ proteins during $\mathrm{G}_{1} / \mathrm{S}$. Donovan and J. Diffley, unpubl.)

Together, these experiments provide a tangible link between yeast pre-RCs and Xenopus licensing and suggest that this origin resetting pathway is a conserved feature in eukaryotic DNA replication.

(Step 2) S phase promotion: competence for origin firing and a block to pre-RC formation

Once assembled, pre-RCs become activated during $\mathrm{S}$ phase through the action of at least two different protein kinases. In budding yeast these kinases are encoded by the CDC7 and CDC28 genes.

$C d c 7 p$ Early genetic analysis indicated that Cdc7p plays an essential role at the $\mathrm{G}_{1}-\mathrm{S}$ transition (Hereford and Hartwell 1974). The targets of Cde7p phosphoryla- tion required to trigger origin firing are currently unknown; however, evidence suggests that these targets are likely to be components of initiation complexes. The Cdc $7 p$ kinase requires an additional protein, Dbf4p, for its activity (Chapman and Johnston 1989; Kitada et al. 1992; Jackson et al. 1993). When Db4p is fused to the transcriptional activation domain of the GAL4 protein, it can activate transcription of the lac $Z$ reporter when a replication origin is cloned upstream of lacZ. This interaction with replication origins in a one-hybrid assay is dependent on an intact ACS, suggesting that the interaction of Dbf4p with origins is not direct but occurs through ORC (Dowell et al. 1994). Whether this interaction is direct with ORC or indirect through another protein is not known. Consistent with the possibility that $\mathrm{ORC}$ and $\mathrm{Cdc} 7 \mathrm{p}$ may interact in vivo is the fact that $c d c 7$ mutants exhibit synthetic lethality with orc2 mutants (Loo et al. 1995; Hardy 1996) and ORC2 and Cdc7p interact in two-hybrid experiments (Hardy 1996). Dbf4p also interacts with Cdc7p (Kitada et al. 1992; Jackson et al. 993); however, different domains of Dbf4p are required for origin interaction and $\mathrm{Cdc} 7 \mathrm{p}$ interaction, suggesting that $\mathrm{Dbf} 4 \mathrm{p}$ may act to recruit $\mathrm{Cdc} 7 \mathrm{p}$ to origins (Dowell et al. 1994).

Cdc28p CDC28 encodes the major cyclin-dependent kinase $(\mathrm{Cdk})$ in budding yeast. The cyclin subunits of this kinase in budding yeast fall, broadly, into two phylogenetic groups: the $\mathrm{G}_{1}$ cyclins (Clns) and the B-type cyclin (Clbs). The $\mathrm{G}_{1}$ cyclins are primarily involved in regulating the commitment to proliferate (Start), whereas the Clbs, as in higher eukaryotes, are essential for entry into mitosis. In addition, cells in which all six Clbs have been depleted cannot enter $S$ phase after Start, implying an essential role for $\mathrm{Cdc} 28 \mathrm{p}$ in promoting DNA synthesis at the $G_{1}-S$ boundary (Schwob et al. 1994). Therefore, in budding yeast, $\mathrm{Cdc} 28 \mathrm{p}$ has two distinct roles in $\mathrm{G}_{1}$, one involves the Clns at Start and the second involves the Clbs at the $G_{1}-S$ transition. Cln-Cdc28p kinase seems to be required primarily for the development of $\mathrm{Clb}-$ Cdc28p kinase (Schneider et al. 1996; Tyers 1996), whereas the role of the Clb kinase in initiating DNA replication is less clear.

Experiments with Xenopus cell-free extracts have been important in demonstrating and characterizing the essential role for Cdks in initiating DNA replication. Depletion of extracts with either p13 $3^{\text {SUC1 }}$ beads (Blow and Nurse 1990) or with antibodies to Cdk2 (Fan and Newport 1991) blocks DNA replication. Replication can be restored to differing extents by addition of either recombinant cyclin protein (cyclin E or cyclin A) or mRNA encoding Cdc2 or Cdk2 (Chevalier et al. 1995; Jackson et al. 1996; Strausfeld et al. 1996). Together, the frog and yeast experiments suggest that an essential role for Cdks in the firing of replication origins may be a common feature of all eukaryotic cell types.

In fission yeast, the major $\mathrm{Cdk}$ is encoded by $c d c 2^{+}$ and the single essential mitotic cyclin is encoded by 
$c d c 13^{+}$. An important series of expriments in this organism has provided several lines of evidence indicating that the mitotic Cdks have an additional role in blocking rereplication during the cell cycle. First, temperaturesensitive $c d c 2^{+}$mutants can be induced to undergo an additional round of DNA replication at the restrictive temperature (Broek et al. 1991). Second, deletion of $c d c 13^{+}$leads to endoreduplication (Hayles et al. 1994). And third, overexpression of the Cdk inhibitor, rum $1^{+}$ (Correa Bordes and Nurse 1995), also leads to endoreduplication (Moren and Nurse 1994). This appears to be true in Drosophila as well because loss of mitotic cyclins during embryogenesis leads to generation of polyploid cells (Lehner and O'Farrell 1990; Sauer et al. 1995).

Mitotic cyclin-cdk activity blocks rereplication in budding yeast as well; transient inhibition of $\mathrm{Clb}$ Cdc28p kinase in $\mathrm{G}_{2}$-blocked cells caused by transient expression of the Cdk inhibitor $\mathrm{p} 40^{\text {SIC1 }}$ promotes an additional round of DNA replication (Dahmann et al. 1995). Experiments in budding yeast have extended this idea in three important ways. In budding yeast, there are six B type cyclins, Clb1-Clb6 /Ghiara et al. 1991; Surana et al. 1991; Epstein and Cross 1992; Fitch et al. 1992; Richardson et al. 1992; Kuhne and Linder 1993; Schwob and Nasmyth 1993). Normally Clb5 and Clb6 function to promote passage through $\mathrm{S}$ phase, whereas $\mathrm{Clbl}-\mathrm{Clb} 4$ function later in the cell cycle to promote entry into mitosis. However, in the absence of the $S$ phase-promoting Clbs, the mitotic Clbs promote $\mathrm{G}_{1}$ cells into $\mathrm{S}$ phase, not $M$ phase (Epstein and Cross 1992; Kuhne and Linder 1993; Schwob and Nasmyth 1993). Therefore, the state of the cell dictates, at least in part, its response to $\mathrm{Clb}$ kinase.

In the absence of the mitotic Clbs (Clb1-Clb4), the S phase Clbs (Clb5 and Clb6) are sufficient to block rereplication. Cells blocked in $\mathrm{G}_{2} / \mathrm{M}$ by depletion of ClblClb4 can still be induced to undergo rereplication by transient $\mathrm{p} 40^{S I C 1}$ expression. Moreover, several mutants that allow rereplication to occur when Clbl-Clb4 have been depleted appear to act by lowering levels of the remaining Clbs (Clb5 and Clb6) (Dahmann et al. 1995). Therefore, both the $M$ and the $S$ phase-promoting cyclins can block rereplication. The important implication from these experiments is that both the $S$ phase and $M$ phase $\mathrm{Clb}$ kinases can play a dual role in regulating replication: firing origins in $\mathrm{S}$ phase and blocking rereplication until the next cell cycle.

Finally, experiments in budding yeast have indicated that Clb kinases block rereplication by blocking the formation of Cdc6p-dependent pre-RCs. Again by exploitation of strains in which the CDC6 gene is under the control of regulatable promoters, the ability of Cdc $6 \mathrm{p}$ to form pre-RCs at different times during the cell cycle was examined (Piatti et al. 1996). These experiments showed that Cdc6p synthesis can drive pre-RC formation during $\mathrm{G}_{1}$; however, if cells are allowed to proceed deeper into the cell cycle in the absence of Cdc6p, de novo Cdc6p synthesis is no longer sufficient to induce pre-RC formation. Thus, there exists a "point of no return," after which Cdc6p cannot induce pre-RC formation and, therefore, cannot induce DNA replication. This point of no return can be delayed by elimination of Clb5 and Clb6 proteins, consistent with the notion that these Clbs can block replication and, presumably, rereplication, by a blocking pre-RC formation. This notion is supported by a second set of experiments showing that expression of the Clb kinase inhibitor $\mathrm{p} 40^{S I C 1}$ in $\mathrm{G}_{2}$ is sufficient to promote the inappropriate formation of pre-RCs (Dahmann et al. 1995).

\section{Conclusions}

The skeleton of a biochemical pathway has emerged from the studies described above. The first step in the pathway is the binding of ORC to origins of replication. In budding yeast, this occurs immediately after initiation in the previous cell cycle and involves recognition of a specific DNA sequence within replicators. ORC remains bound at budding yeast origins during the entire cell cycle as well as during periods of quiescence. Much remains to be learned about the timing and specificity of origin recognition by ORC in higher eukaryotes. ORC binding precedes and is required for the Cdc6p-dependent assembly of pre-RCs and recruitment of the $\mathrm{Mcm}$ proteins to prereplicative chromatin. The protein components of pre-RCs are currently unknown. Cdc6p may be a component of these complexes or it may act as an assembly factor, loading other proteins such as the $\mathrm{Mcm}$ proteins into these complexes. The roles of these proteins in inducing origin unwinding and in recruiting the DNA synthesis machinery have yet to be addressed.

Clb kinases play a dual role-triggering the activation of existing pre-RCs and blocking the formation of new preRCs. Cdc6p itself is a good candidate for being the target of inhibition of pre-RC formation by Clb kinase, as complexes between Cdc6p and Clb kinases have been identified in cell extracts (Piatti et al. 1996). Additionally, overexpression of the fission yeast Cdc6p homolog, $c d c 18^{+}$, induces endoreduplication (Nishitani and Nurse 1995; Muzi-Falconi et al. 1996). Although it is not clear how this occurs, one possibility is that $c d c 18^{+}$protein expression levels exceed the capacity of the Clb kinases to block their action in pre-RC formation. Jallepalli and Kelly have recently shown that overproduction of rum $1^{+}$leads to the accumulation of $c d c 18^{+}$protein (Jallepalli and Kelly 1996), suggesting that Clb kinases may act to destabilize the $c d c 18^{+}$protein.

Little is also known about how Clb-Cdc28 and Dbf4 Cdc7 kinases trigger initiation. Clearly, identification of the physiologically relevant targets of these kinases is critical. The activation of these kinases at the $\mathrm{G}_{1}-\mathrm{S}$ transition establishes a state of competence for origin firing; however, all origins do not fire synchronously at this point. Why the firing of some origins is delayed is currently unclear (Fangman and Brewer 1992) and further work is required to understand the temporal structure of $S$ phase.

A model for cell-cycle regulation of DNA replication based on the results described in this review is shown in Figure 2. The destruction of Clb kinases at the end of 


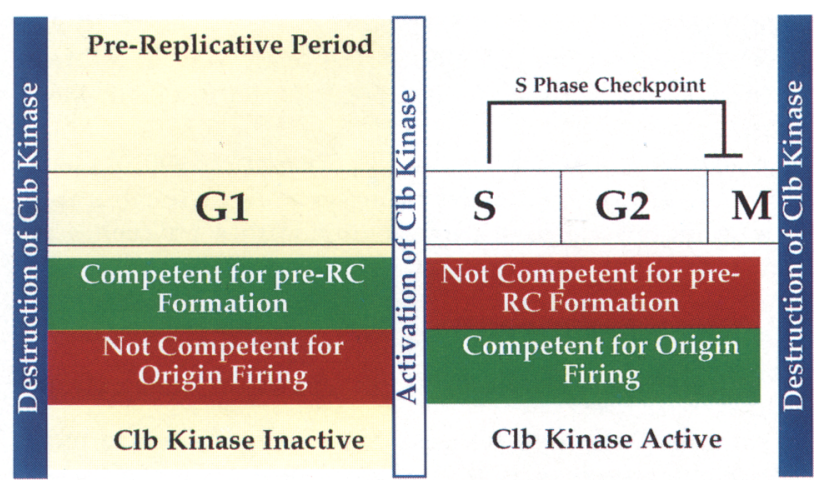

Figure 2. Oscillations in $\mathrm{Clb}$ kinase activity couple mitosis and $\mathrm{S}$ phase. The cell cycle begins with the destruction of $\mathrm{Clb}$ kinases at the end of mitosis. During the subsequent prereplicative period cells are competent to assemble pre-RCs at replication origins but not to activate these complexes and initiate DNA replication. Environmental signals such as growth factors and internal cues such as cell size and genome integrity contribute to the cascade of $G_{1}$ events culminating in the development of active $\mathrm{Clb}$ kinase. The activation of $\mathrm{Clb}$ kinase ends the prereplicative period. Cells are now competent to activate pre$\mathrm{RCs}$ and initiate DNA replication but are no longer competent to form new pre-RCs. Some of the possible mechanisms for ordering $S$ and $M$ phases are discussed in the text and include the S-phase checkpoint shown in the figure.

mitosis is one of the most significant moments in the cell cycle, marking the beginning of the prereplicative period. Because of the lack of Clb kinase activity, preRCs are free to form at any time during the prereplicative period. The actual timing of pre-RC formation may vary from cell type to cell type depending on requirements. For example, embryonic cells or rapidly proliferating yeast cells assemble pre-RCs as soon as cells enter $\mathrm{G}_{1}$. Alternatively, slower growing cells and cells that remain quiescent for long periods of time may assemble pre-RCs later in $G_{1}$. The regulation of expression of key proteins such as Cdc6p is likely to be critical in determining patterns of pre-RC formation in $G_{1}$.

The commitment to proliferate initiates the cascade of events leading to the activation of $\mathrm{Clb}$ kinases. This marks the end of the prereplicative period. As described above, a single $\mathrm{Clb}$ kinase can cause cells to enter $\mathrm{S}$ phase from $G_{1}$ or to enter mitosis from $G_{2}$. Thus, the state of a cell determines its response to $\mathrm{Clb}$ kinase, and in this model, it is the presence or absence of pre-RCs that determines the response cells will have to $\mathrm{Clb} \mathrm{ki}$ nases. This explains why $G_{1}$ cells enter mitosis but not $S$ phase in the absence of Cdc6p or $c d c 18^{+}$protein land, hence pre-RCs). It does not explain why mitosis does not start at the same time as $\mathrm{S}$ phase. In fact there are probably a number of ways that cells normally delay entry into mitosis until $\mathrm{S}$ phase is complete. For example, as described above, all Clbs are not equivalent. Although the mitotic cyclins such as Clb1-Clb4 in budding yeast and the $c d c 13^{+}$gene product in fission yeast can drive $S$ phase, the S-phase cyclins such as Clb5 and Clb6 and $\mathrm{cig}^{+}$and $\mathrm{cig} 2^{+}$cannot drive entry into mitosis. Because
Clb5 and Clb6 are expressed well before Clb1-Clb4, for example, a simple timing mechanism ensures that $S$ phase will begin before mitosis. The reason for the inability of the S-phase cyclins to promote mitosis is unknown but may reflect an inability to recognize and phosphorylate some critical target proteins.

The actual levels of Clb kinase may also be critical; low levels favoring entry into $\mathrm{S}$ phase and high levels favoring entry into mitosis (Stern and Nurse 1996). A particularly striking example of this is the response that sucl-depleted frog extracts have to addition of cyclin A (Strausfeld et al. 1996); low levels promote entry into S phase, whereas high levels do not promote DNA synthesis but, rather, cause chromosome condensation and nuclear envelope breakdown such as that seen on entry into mitosis.

In addition, most cells have a signal transduction pathway that senses the presence of unreplicated DNA and, through the action of several protein kinases, blocks entry into mitosis (Hartwell and Weinert 1989; Murray 1992; Weinert and Lydall 1993; Elledge and Harper 1994). Such checkpoints are critical in establishing an actual dependency; cells cannot enter mitosis until DNA synthesis is complete.

Finally, the mitotic cyclins not only drive entry into mitosis, but they also trigger their own destruction at the end of mitosis by activating the anaphase promoting complex (Murray 1995). This returns cells to the beginning of a new cell cycle and a new prereplicative period.

Over the past several years, a great deal has been learned about the factors involved in initiating eukaryotic DNA replication and their regulation in global terms. Much remains to be learned, however, about the molecular details involved in both the regulation and the actual mechanism of initiation. Thus, we can anticipate a great deal of further excitement in the field over the next several years.

\section{Acknowledgments}

I thank members of my lab for many helpful discussions and William Dunphy and Julian Blow for commmunicating results prior to publication.

\section{References}

Aladjem, M.I., M. Groudine, L.L. Brody, E.S. Dieken, R.E.K. Fournier, G.M. Wahl, and E.M. Epner. 1995. Participation of the human $\beta$-globin locus control region in initiation of DNA replication. Science 270: 815-819.

Bell, S.P. and Stillman, B. 1992 Nucleotide dependent recognition of chromosomal origins of DNA replication by a multiprotein complex. Nature 357: 128-134.

Bell, S.P., R. Kobayashi, and B. Stillman. 1993. Yeast origin recognition complex functions in transcription silencing and DNA replication. Science 262: 1844-1849.

Bell, S.P., J. Mitchell, J. Leber, R. Kobayashi, and B. Stillman. 1995. The multidomain structure of Orclp reveals similarity to regulators of DNA replication and transcriptional silencing. Cell 83: 563-568.

Blow, J.J. and R.A. Laskey. 1986. Initiation of DNA replication 
in nuclei and purified DNA by a cell-free extract of Xenopus eggs Cell 47: 577-587.

- 1988. A role for the nuclear envelope in controlling DNA replication within the cell cycle. Nature 332: 546-548.

Blow, J.J. and P. Nurse. 1990. A cdc2-like protein is involved in the initiation of DNA replication in Xenopus egg extracts. Cell 62: 855-862.

Blumenthal, A.B., H.J. Kriegstein, an D.S. Hogness. 1973. The units of DNA replication in Drosophila melanogaster chromosomes. Cold Spring Harbor Symp. Quant. Biol. 38: 205223.

Borowiec, J.A., F.B. Dean, P.A. Bullock, and J. Hurwitz. 1990. Binding and unwinding-how $\mathrm{T}$ antigen engages the SV40 origin of DNA replication. Cell 60: 181-184.

Bramhill, D. and A. Kornberg. 1988. A model for initiation at origins of DNA replication. Cell 54: 915-918.

Brewer, B.J. and W.L. Fangman. 1987. The localization of replication origins on ARS plasmids in $S$. cerevisiae. Cell 51: 463-471.

Broek, D., R. Bartlett, K. Crawford, and P. Nurse. 1991. Involvement of $\mathrm{p} 43^{c d c 2}$ in establishing the dependency of $S$ phase on mitosis. Nature 349: 388-393.

Cairns, J. 1966. Autoradiography of HeLa cell DNA. J. Mol. Biol. 15: 373-373.

Campbell, J.L. and C.S. Newlon. 1991. Chromosomal DNA replication. In The molecular biology of the yeast Saccharomyces: Genome dynamics, protein synthesis and energetics |ed. J.R. Broach, J.R. Pringle, and E.W Jones), pp. 41-146. Cold Spring Harbor Laboratory Press, Cold Spring Harbor, NY.

Carpenter, P.B., P.R. Mueller, and W.G. Dunphy. 1996. Role for a Xenopus Orc2-related protein in controlling DNA replication. Nature 379: 357-360.

Challberg, M.D. aned T.J. Kelly. 1989. Animal virus DNA replication. Annu. Rev. Biochem. 58: 671-717.

Chapman, J.W. and L.H. Johnston. 1989. The yeast gene, DBF4, essential for entry ino $S$ phase is cell cycle regulated. Exp. Cell. Res. 180: 419-428.

Chevalier, S., J.P. Tassan, R. Cox, M. Philippe, and C. Ford. 1995. Both cdc2 and cdk2 promote S-phase initiation in Xenopus egg extracts. J. Cell Sci. 108: 1831-1841.

Chong, J.P.J., H.M. Mahbubani, C.-Y. Khoo, and J.J. Blow. 1995. Purification of an MCM-containing complex as a component of the DNA replication licensing system. Nature 375: 418421.

Chong, J.P.J., P. Thömmes, and J.J. Blow. 1996. The role of $\mathrm{MCM} / \mathrm{Pl}$ proteins in the licensing of DNA replication. Trends Biochem. Sci. 21: 102-106.

Cocker, J.H. 1995. "Protein-DNA interactions at yeast replication origins in vivo." Ph.D. thesis, Imperial College of Science, Technology and Medicine, University of London, London, UK.

Cocker, J.H., S. Piatti, C. Santocanale, K. Nasmyth, and J.F.X. Diffley. 1996. An essential role for the Cdc6 protein in forming the pre-replicative complexes of budding yeast. Nature 379: $180-182$.

Coleman, T.R., P.B. Coleman, and W.G. Dunphy. 1996. The Xenopus Cdc6 protein is essential for the initiation of a single round of DNA replication in cell-free extracts. Cell (in press).

Correa Bordes, J. and P. Nurse. 1995. p25 $5^{\text {ruml }}$ orders S phase and mitosis by acting as an inhibitor of the $\mathrm{p} 34^{\text {cdc2 }}$ mitotic kinase. Cell 83: 1001-1009.

Coxon, A., K. Maundrell, and S.E. Kearsey. 1992. Fission yeast $c d c 21+$ belongs to a family of proteins involved in an early step of chromosome-replication. Nucleic Acids Res.
20: $5571-5577$.

Crevel, G. and S. Cotterill. 1991. DNA-replication in cell-freeextracts from Drosophila melanogaster. EMBO I. 10: 43614369.

Dahmann, C., J.F.X Diffley, and K. Nasmyth. 1995. S-phasepromoting cyclin-dependent kinases prevent re-replication by inhibiting the transition of origins to a pre-replicative state Cur. Biol. 5: 1257-1269.

Dalton, S. and L. Whitbread. 1995. Cell-cycle-regulated nuclear import and export of $\mathrm{Cdc} 47$, a protein essential for initiation of DNA-replication in budding yeast. Proc. Natl Acad. Sci. 92: 2514-2518.

Depamphilis, M.L. 1993. Origins of DNA-replication in metazoan chromosomes. J. Biol. Chem. 268: 1-4.

Diffley, J.F.X. 1992. Early events in eukaryotic DNA replication. Trends Cell Biol. 2: 298-303.

1995. The initiation of DNA replication in the budding yeast cell division cycle. Yeast 11: 1651-1670.

Diffley, J.F.X. and J.H. Cocker. 1992. Protein-DNA interactions at a yeast replication origin. Nature 357: 169-172.

Diffley, J.F.X., J.H. Cocker, S.J. Dowell, and A. Rowley. 1994. Two steps in the assembly of complexes at yeast replication origins in vivo. Cell 78: 303-316.

Dowell, S.J., P. Romanowski, and J.F.X. Diffley. 1994. Interactions of $\mathrm{Dbf} 4$, the $\mathrm{Cdc} 7$ protein kinase regulatory subunit, with yeast replication origins in vivo. Science 265: 12431246.

Ehrenhofer Murray, A.E., M. Gossen, D.T. Pak, M.R. Botchan, and J. Rine. 1995. Separation of origin recognition complex functions by cross-species complementation. Science 270: $1671-1674$.

Elledge, S.J. and J.W. Harper. 1994. Cdk inhibitors: On the threshold of checkpoints and development. Curr. Opin. Cell. Biol. 6: 847-852.

Epstein, C.B. and F.R. Cross. 1992. CLB5: A novel B cyclin from budding yeast with a role in S phase. Genes \& Dev. 6: 16951706.

Fang, F. and J.W. Newport. 1991. Evidence that the G1-S and G2-M transitions are controlled by different $\mathrm{Cdc} 2$ proteins in higher eukaryotes. Cell 66: 731-742.

Fangman, W.L. and B.J. Brewer. 1992. A question of time-replication origins of eukaryotic chromosomes. Cell 71:363366.

Fitch, I., C. Dahmann, U. Surana, A. Amon, K. Nasmyth, L. Goetsch, B. Byers, and B. Futcher. 1992. Characterization of four B-type cyclin genes of the budding yeast Saccharomyces cerevisiae. Mol. Biol. Cell. 3: 805-818.

Forsburg, S.L. and P. Nurse. 1994. The fission yeast $\operatorname{cdcl} 9^{+}$gene encodes a member of the MCM family of replication protein. I. Cell. Sci. 107: 2779-2788.

Foss, M., F.J. McNally, P. Laureson, and J Rine. 1993. Origin recognition complex (ORC) in transcriptional silencing and DNA replication in S. cerevisiae. Science 262: 1838-1844.

Fox, C.A., S. Loo, A. Dillin, and J. Rine. 1995. The origin recognition complex has essential functions in transcriptional silencing and chromosomal replication. Genes \& Dev. 9: 911-924.

Gavin, K.A., M. Hidaka, and B. Stillman. 1995. Conserved initiator proteins in eukaryotes. Science 270: 1667-1671.

Ghiara, J.B., H.E. Richardson, K. Sugimoto, M. Henze, D.J. Lew, C. Wittenberg, and S.I. Reed. 1991. A cyclin B homolog in S. cerevisiae: Chronic activation of the $\mathrm{Cdc} 28$ protein kinase by cyclin prevents exit from mitosis. Cell 65: 163-174.

Gossen, M. D.T. Pak, S.K. Hansen, J.K. Acharya, and M. Botchan. 1995. A Drosophila homolog of the yeast origin recognition complex. Science 270: 1674-1677. 
Haase, S.B., S.S. Heinzel, and M.P. Calos. 1994. Transcription inhibits the replication of autonomously replicating plasmids in human-cells. Mol. Cell. Biol. 14: 2516-2524.

Hardy, C. 1996. Characterization of an essential Orc2p-associated factor that plays a role in DNA replication. Mol. Cell. Biol. 16: 1832-1841.

Harland, R. 1981. Initiation of DNA replication in eukaryotic chromosomes. Trends Biochem. Sci. 6: 71-74.

Harland, R.M. and R.A. Laskey. 1980. Regulated replication of DNA micro-injected into eggs of Xenopus laevis. Cell 21: 761-771.

Hartwell, L.H. 1976. Sequential function of gene products relative to DNA synthesis in the yeast cell cycle. I. Mol. Biol. 104: 803-817.

Hartwell, L.H. and T.A. Weinert. 1989. Checkpoints: Controls that ensure the order of cell cycle events. Science 246: 629634.

Hayles, J., D. Fisher, A. Woolard, and P. Nurse. 1994. Temporal order of $\mathrm{S}$ phase and mitosis in fission yeast is determined by the state of the $\mathrm{p} 34^{\mathrm{cdc} 2}$-mitotic B cyclin complex. Cell 78: 813-822.

Hennessey, K.M., A. Lee, E. Chen, and D. Botstein. 1991. A group of interacting yeast DNA replication genes. Genes \& Dev. 5: 958-969.

Hereford, L.M. and L.H. Hartwell. 1974. Sequential gene function in the initiation of Saccharomyces cerevisiae DNA synthesis. J. Mol. Biol. 84: 445-461.

Hogan, E. and D. Koshland. 1992. Addition of extra origins of replication to a minichromosome suppresses its mitotic loss in $c d c 6$ and cdc14 mutants of Saccharomyces cerevisiae. Proc. Natl. Acad. Sci. 89: 3098-3102.

Hori, Y., K. Shirahige, C. Obuse, T. Tsurimoto, and $\mathrm{H}$. Yoshikawa. 1996. Characterization of a novel $C D C$ gene (ORC1) partly homologous to CDC6 of Saccharomyces cerevisiae. Mol. Biol. Cell. 7: 409-418.

Huang, R.Y. and D. Kowalski. 1996., Multiple DNA elements in ARS305 determine replication origin activity in a yeast chromosome. Nucleic Acids Res. 24: 816-823.

Huberman, J.A. and A.D. Riggs. 1966. Autoradiography of chromosomal DNA fibers from Chinese hamster cells. Proc. Natl. Acad. Sci. 55: 599-606.

. 1968. On the mechanism of DNA replication in mam malian chromosomes. I. Mol. Biol. 32: 327-341.

Huberman, J.A., L.D. Spotila, K.A. Nawotka, S.M. El Assouli, and L.R. Davis. 1987. The in vivo replication origin of the yeast 2 micron plasmid. Cell 51: 473-481.

Hyrien, O. and M. Mechali., 1992. Plasmid replication in Xenopus eggs and egg extracts-a 2D gel-electrophoretic analysis. Nucleic Acids Res. 20: 1463-1469.

Hyrien, O., C. Maric, and M. Mechali. 1995. Transition in specification of embryonic metozoan DNA replication origins. Science 270: 994-997.

Jackson, A.L., P.M. Pahl, K. Harrison, J. Rosamond, and R.A. Sclafani. 1993. Cell cycle regulation of the yeast CDC7 protein kinase by association with the DBF4 protein. Mol. Cell. Biol. 13: 2899-2908.

Jackson, P.K., S. Chevalier, M. Philippe, and M.W. Kirschner. 1996. Early events in DNA-replication require cyclin-E and are blocked by p21 cipl. J. Cell Biol. 130: 755-769.

Jacob, F., S. Brenner, and F. Cuzin. 1963. On the regulation of DNA replication in bacteria. Cold Spring Harbor Symp. Quant. Biol. 28: 329-348.

Jallepalli, P.V. and T.J. Kelly. 1996. Rum 1 and Cdc18 link inhibition of cyclin-dependent kinase to the initiation of DNA replication in Schizosaccharomyces pombe. Genes \& Dev. 10: $541-552$.
Kelly, T.J. 1988. SV40 DNA replication. I. Biol. Chem. 263: $17889-17892$.

Kelly, T.J., G.S. Martin, S.L. Forsburg, R.J. Stephen, A. Russo, and P. Nurse. 1993. The fission yeast $c d c 28^{+}$gene product couples S phase to START and mitosis. Cell 74: 371-382.

Kitada, K., L.H. Johnston, T. Sugino, and A. Sugino. 1992. Temperature-sensitive $c d c 7$ mutations of Saccharomyces cerevisiae are suppressed by the $D B F 4$ gene, which is required for the G1/S cell cycle transition. Genetics 131: 21-29.

Kitsberg, D., S. Selig, I. Keshet, and H. Cedar. 1993. Replication structure of the human $\beta$-globin gene domain. Nature 366: $588-590$.

Koonin, E.V. 1993. A common set of conserved motifs in a vast variety of putative nucleic acid-dependent ATPases including MCM proteins involved in the initiation of eukaryotic DNA replication. Nucleic Acids Res. 21: 2541-2547.

Krysan, P.J., J.G. Smith, and M.P. Calos. 1993 Autonomous replication in human cells of multimers of specific human and bacterial DNA sequences. Mol. Cell. Biol.13: 2688-2696.

Kubota, Y., S. Mimura, S.-I. Nishimoto, H. Takisawa, and H. Nojima. 1995. Identification of the yeast MCM3-related protein as a component of Xenopus DNA replication licensing factor. Cell 81: 601-609.

Kuhne, C. and P. Linder. 1993. A new pair of B-type cyclins from Saccharomyces cerevisiae that function early in the cell cycle. $E M B O$ J. 12: 3437-3447.

Leatherwood, I., A. Lopez Girona, and P. Russell. 1996. Interaction of Cdc 2 and $\mathrm{Cdc} 18$ with a fission yeast ORC2-like protein. Nature 379: 360-363.

Lehner, C.F. and P.H. O'Farrell. 1990. The roles of Drosophila cyclins A and B in mitotic control. Cell 61: 535-547.

Li, J.J. and I. Herskowitz. 1993. Isolation of ORC6, a component of the yeast origin recognition complex by a one-hybrid system. Science 262: 1870-1874.

Liang, C., M. Weinreich, and B. Stillman. 1995. ORC and Cdc6p interact and determine the frequency of initiation of DNA replication in the genome. Cell 81: 667-676.

Lohka, M.J. ad Y. Masui. 1983. Formation in vitro of sperm pronuclei and mitotic chromosomes induced by amphibian ooplasmic components. Science 220: 719-721.

Loo, S., C.A. Fox, J. Rine, R. Kobayashi, B. Stillman, and S. Bell. 1995. The origin recognition complex in silencing, cell-cycle progression, and DNA-replication. Mol. Cell. Biol. 6: 74l756.

Madine, M.A., C.-Y. Khoo, A.D. Mills, and R.A. Laskey. 1995a. MCM3 complex required for cell cycle regulation of DNA replication in vertebrate cells. Nature 375: 421-424.

Madine, M.A., C.-Y. Khoo, A.D. Mills, C. Mushal, and R.A. Laskey. 1995b. The nuclear envelope prevents reinitiation of replication by regulating the binding of MCM3 to chromatin in Xenopus egg extracts. Curr. Biol. 5: 1270-1279.

Mahbubani, H.M., T. Paull, J.K. Elder, and J.J. Blow. 1992. DNA replication initiates at multiple sites on plasmid DNA in Xenopus egg extracts. Nucleic Acids Res. 20: 1457-1462.

Maine, G.T., P. Sinha, and B.-K. Tye. 1984. Mutants of S. cerevisiae defective in the maintenance of minichromosomes. Genetics 106: 365-385.

Marahrens, Y. and B. Stillman. 1992. A yeast chromosomal origin of DNA replication defined by multiple functional elements. Science 255: 817-823.

Micklem, G., A. Rowley, J. Harwood, K. Nasmyth, and J.F.X. Diffley. 1993. Yeast origin recognition complex is involved in DNA replication and transcriptional silencing. Nature 366: $87-89$.

Miyake, S., N. Okishio, I. Samejima, Y. Hiraoka, T. Toda, I. Saitoh, and M. Yanagia. 1993. Fission yeast genes $n d a 1^{+}$and 
nda $4^{+}$, mutations of which lead to S-phase block, chromatin alteration and $\mathrm{Ca}^{2+}$ suppression, are members of the Cdc46/ Mcm2 family. Mol. Biol. Cell. 4: 1003-1015.

Moir, D., S.E. Stewart, B.C. Osmond, and D. Botstein. 1982. Cold-sensitive cell-division-cycle mutants of yeast: Isolation, properties, and pseudoreversion studies. Genetics 100: $547-563$.

Moreno, S. and P. Nurse. 1994. Regulation of progression through the G1 phase of the cell-cycle by the rum $1^{+}$gene. Nature 367: 236-242.

Murray, A. 1995. Cyclin ubiquitination-the destructive end of mitosis. Cell 81: 149-152.

Murray, A.W. 1992. Creative blocks-cell-cycle checkpoints and feedback controls. Nature 359: 599-604.

Muzi-Falconi, M. and T.J. Kelly. 1995. Orp1, a member of the Cdc18/Cdc6 family of S-phase regulators, is homologous to a component of the origin recognition complex. Proc. Natl. Acad. Sci. 92: 12475-12479.

Muzi-Falconi, M., G.W. Brown, and T.J. Kelly. 1996. Cdc18+ regulates initiation of DNA replication in Schizosaccharomyces pombe. Proc. Natl. Acad. Sci. 93: 1566-1570.

Nishitani, H. and P. Nurse. 1995. p65 $5^{\text {cdc18 }}$ plays a major role controlling the initiation of DNA replication in fission yeast. Cell 83: 397-405.

Piatti, S., C. Lengauer, and K. Nasmyth. 1995. Cdc6 is an unstable protein whose de novo synthesis in Gl is important for the onset of S phase and for preventing a "reductional" anaphase in the budding yeast Saccharomyces cerevisiae. EMBO J. 14: 3788-3799.

Piatti, S., T. Bohm, J.H. Cocker, J.F.X. Diffley, and K. Nasmyth. 1996. Activation of S-phase promoting CDKs in late $G_{1}$ defines a "point of no return" after which Cdc6 synthesis cannot promote DNA replication in yeast. Genes \& Dev. 10: $1516-1531$.

Rao, H. and B. Stillman. 1995. The origin recognition complex interacts with a bipartite DNA binding site within yeast replicators. Proc. Natl. Acad. Sci. 92: 2224-2228.

Rao, H., Y. Marahrens, and B. Stillman. 1994. Functional conservation of multiple elements in yeast chromosomal replicators. Mol. Cell. Biol. 14: 7643-7651.

Rao, P.N. and R.T. Johnson. 1970. Mammalian cell fusion. Studies on the regulation of DNA synthesis and mitosis. Nature 225: 159-164.

Richardson, H., D.J. Lew, M. Henze, K. Sugimoto, and S.I. Reed. 1992. Cyclin-B homologs in Saccharomyces cerevisiae function in $S$ phase and in $G_{2}$. Genes \& Dev. 6: 2021-2034.

Rowles, A., J.P.J. Chong, L. Brown, M. Howell, G.I. Evan, and J.J. Blow. 1996. Interaction between the origin recognition complex and the replication licensing system in Xenopus. Cell (in press).

Rowley, A., J.H. Cocker, J. Harwood, and J.F.X. Diffley. 1995. Initiation complex assembly at budding yeast replication origins begins with the recognition of a bipartite sequence by limiting amounts of the initiator, ORC. EMBO J. 14: 26312641.

Sauer, K., J.A. Knoblich, H. Richardson, and C.F Lehner. 1995. Distinct modes of cyclin $\mathrm{E} / \mathrm{cdc} 2$ kinase regulation and S-phase control in mitotic and endoreduplication cycles of Drosophila embryogenesis. Genes \& Dev. 9: 1327-1339.

Schneider, B.L., Q.-H. Yang, and A.B. Futcher. 1996. Linkage of replication to start by the cdk inhibitor Sicl. Science 272: $560-562$.

Schwob, E. and K. Nasmyth. 1993. CLB5 and CLB6: A new pair of B cyclins involved in DNA replication in Saccharomyces cerevisiae. Genes \& Dev. 7: 1160-1175.

Schwob, E., T. Bohm, M.D. Mendenhall, and K. Nasmyth. 1995.
The B-type cyclin kinase inhibitor $\mathrm{p} 40^{\mathrm{SIC} 1}$ controls the G1 to $\mathrm{S}$ transition in S. cerevisiae. Cell 79: 233-244.

Snyder, M., R. Sapolsky, and R.W. Davies. 1988. Transcription interferes with elements important for chromosome maintenance in Saccharomyces cerevisiae. Mol. Cell. Biol. 8: 2184-2194.

Stern, B. and P. Nurse. 1996. A quantitative model for the cdc2 control of $\mathrm{S}$ phase and mitosis in fission yeast. Trends Genet. 12: $345-350$.

Stillman, B. 1989. Initiation of eukaryotic DNA replication in vitro. Annu. Rev. Cell Biol. 5: 197-245.

1993. DNA-replication-replicator renaissance. Nature 366: 506-507.

Strausfeld, U.P., M. Howell, P. Descombes, S. Chevalier, R.E. Rempel, J. Adamczewski, J.L. Maller, T. Hunt, and J.J. Blow. 1996. Both cyclin-A and cyclin-E have S-phase promoting (SPF) activity in Xenopus egg extracts. J. Cell Sci. 109: 15551563.

Surana, U., H. Robitsch, C. Price, T. Schuster, I. Fitch, A.B. Futcher, and K. Nasmyth. 1991. The role of CDC28 and cyclins during mitosis in the budding yeast $\mathrm{S}$. cerevisiae. Cell 65: 145-161.

Takahashi, K., H. Yamada, and M. Yanagida. 1994. Fission yeast minichromosome loss mutants mis cause lethal aneuploidy and replication abnormality. Mol. Biol. Cell. 5: 1145-1158.

Tanaka, S., D. Halter, M. Livingstone Zatchej, B. Reszel, and F. Thoma. 1994. Transcription through the yeast origin of replication ARS1 ends at the ABFI binding site and affects extrachromosomal maintenance of minichromosomes. $\mathrm{Nu}$ cleic Acids Res. 22: 3904-3910.

Theis, J.F. and C.S. Newlon. 1994. Domain B of ARS307 contains two functional elements and contributes to chromosomal replication origin function. Mol. Cell. Biol. 14: 76527659.

Tyers, M. 1996. The cyclin-dependent kinase inhibitor $\mathrm{p} 40^{\text {sicl }}$ imposes the requirement for Cln G1 cyclin function at Start. Proc. Natl. Acad. Sci. 93: 7772-7776.

Weinert, T. and D. Lydall. 1993. Cell-cycle checkpoints, genetic instability and cancer. Sem. Cancer Biol. 4: 129-140.

Whitbread, L.A. and S. Dalton. 1995. Cdc54 belongs to the $\mathrm{Cdc} 46 / \mathrm{Mcm} 3$ family of proteins which are essential for initiation of eukaryotic DNA-replication. Gene 155: 113-117.

Yan, H., S. Gibson, and B.-K. Tye. 1991. MCM2 and MCM3, two proteins important for ARS activity, are related in structure and function. Genes \& Dev. 5: 944-957.

Yan, H., A.M. Merchant, and B.-K. Tye. 1993. Cell cycle-regulated nuclear localization of MCM2 and MCM3, which are required for the initiation of DNA synthesis at chromosomal replication origins in yeast. Genes \& Dev. 7: 2149-2160.

Zwerschke, W., H.-W. Rottjakob, and H. Küntzel. 1994. The Saccharomyces cerevisiae CDC6 gene is transcribed at late mitosis and encodes a ATP/GTPase controlling S phase initiation. J. Biol. Chem. 269: 23351-23356. 


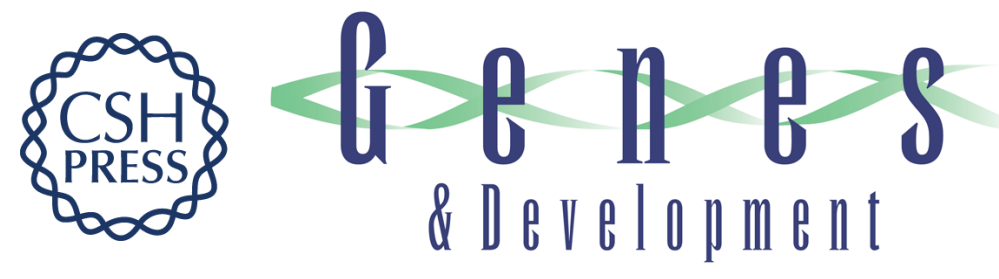

\section{Once and only once upon a time: specifying and regulating origins of DNA replication in eukaryotic cells.}

J F Diffley

Genes Dev. 1996, 10:

Access the most recent version at doi:10.1101/gad.10.22.2819

References This article cites 118 articles, 51 of which can be accessed free at:

http://genesdev.cshlp.org/content/10/22/2819.full.html\#ref-list-1

License

Email Alerting Receive free email alerts when new articles cite this article - sign up in the box at the top Service right corner of the article or click here.

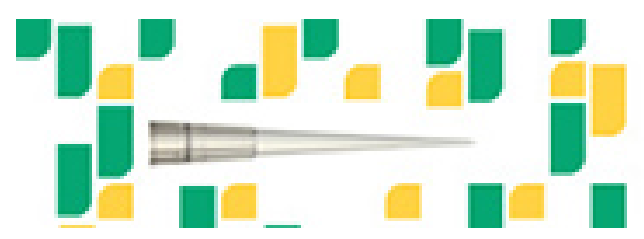

Focused on your science.

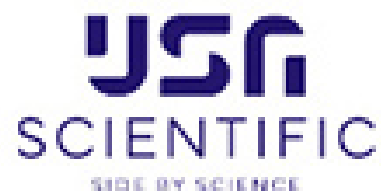

Copyright @ Cold Spring Harbor Laboratory Press 